Meta

Journal des traducteurs

Translators' Journal

\title{
Dérivation suffixale de toponymes : étude d'un terrain propice à la création lexicale
}

\section{Violette Tolédano et Danielle Candel}

Volume 47, numéro 1, mars 2002

URI : https://id.erudit.org/iderudit/007995ar

DOI : https://doi.org/10.7202/007995ar

Aller au sommaire du numéro

\section{Éditeur(s)}

Les Presses de l'Université de Montréal

\section{ISSN}

0026-0452 (imprimé)

1492-1421 (numérique)

Découvrir la revue

\section{Citer cet article}

Tolédano, V. \& Candel, D. (2002). Dérivation suffixale de toponymes : étude d'un terrain propice à la création lexicale. Meta, 47(1), 105-124.

https://doi.org/10.7202/007995ar
Résumé de l'article

Cet article propose l'étude lexicographique d'un ensemble de termes dérivés de toponymes. La plupart d'entre eux ont été repérés dans la presse contemporaine, et relèvent du champ sémantique de « violence » ou de " guerre ". À partir de bases particulièrement évocatrices (les toponymes) et de suffixes (-iser, -ifier -isation, -ification) éminemment dynamiques dans le français actuel, ce mode de formation est fertile dans les domaines historico-géographique et géopolitique, avec des extensions dans les domaines culturels et sociaux. 


\title{
Dérivation suffixale de toponymes: étude d'un terrain propice à la création lexicale
}

\author{
VIOLETTE TOLÉDANO ET DANIELLE CANDEL \\ INaLF, France
}

\begin{abstract}
RÉSUMÉ
Cet article propose l'étude lexicographique d'un ensemble de termes dérivés de toponymes. La plupart d'entre eux ont été repérés dans la presse contemporaine, et relèvent du champ sémantique de «violence» ou de «guerre». À partir de bases particulièrement évocatrices (les toponymes) et de suffixes (-iser, -ifier-isation, -ification) éminemment dynamiques dans le français actuel, ce mode de formation est fertile dans les domaines historico-géographique et géopolitique, avec des extensions dans les domaines culturels et sociaux.
\end{abstract}

\begin{abstract}
This article gives the lexicographical analysis of terms derived from toponyms. Most of them have been evidenced from contemporary newspapers, and are related to the semantic field of "violence" or "war". Each one is built up with a typically dynamic suffix (-iser, -ifier -isation, -ification) and a particularly evocative basis - the toponym. This mode of construction is most fruitful at the crossing of historical and geographical fields, in the geopolitical one, with extensions falling into cultural and social domains as well.
\end{abstract}

\section{MOTS-CLÉS/KEYWORDS}

néologie, toponyme, presse contemporaine, dérivation suffixale (-iser, -isation, -ifier, -ification), géopolitique

\section{Introduction}

Il y a des faits. Il y a eu et il y aura toujours des journalistes pour rendre compte de ces faits - souvent faits de guerre - , que ce soit par écrit ou, comme plus récemment, par l'image et le commentaire télévisuels.

Dire que l'histoire est modelée par les héros (grands capitaines, amiraux, hommes d'exception...) est un truisme, mais déclarer que "L'histoire est toute géographie » pouvait paraître novateur de la part de Michelet dans son Tableau de la France. Il mettait ainsi un accent plus marqué, sinon nouveau, sur le cadre physique dans lequel s'est déroulée la vie des peuples, sur le rôle des frontières naturelles, des montagnes, des fleuves... L'art de la guerre ne s'est-il pas constamment nourri de la connaissance approfondie de la géographie. L'histoire conserve la mémoire référentielle de ces phénomènes géographico-historiques, avec celle des noms de pays où ils se sont déroulés. Notre propos est d'étudier les «retombées» linguistiques du recoupement de la toponymie et de l'histoire, et plus précisément, quelques-uns des nombreux néologismes qui en sont issus. 


\section{D'un nom de lieu à la désignation d'un processus: un cas-type}

\section{I.1. Le rôle de la presse}

Les reporters de la presse écrite, frappés par ces faits "sur le terrain», créent des néologismes à partir de toponymes, symboles du drame aux conséquences d'abord meurtrières, mais aussi sociologiques, géopolitiques. Pour parler d'une situation présentant quelque analogie ou même quelque similarité dans ses conséquences directes avec des lieux, ils opteront plutôt pour tel verbe, telle épithète, tel substantif formés sur une racine qui est un nom de lieu, théâtre de drames récents. De nouvelles lexies sont ainsi apparues pour décrire non seulement le vécu quotidien, mais aussi les analogies frappantes entre le passé et le présent, ou encore pour illustrer d'une manière imagée ou métaphorique des situations antérieures connues de tout un chacun et relevant de l'héritage historique commun. Certaines formations sont, d'autre part, la spécialisation d'un terme déjà existant. Nous constaterons que la création de néologismes remonte, pour certains d'entre eux, à plusieurs décennies, et que dans de nombreux cas il s'agit de couples de néologismes verbe / substantif, ou de l'un ou l'autre isolément.

\section{I.2. Un cas-type: balkaniser, balkanisation}

Un modèle semble s'être créé avec balkaniser. Nous étudierons quelques créations sur ce modèle, avec pour racine un toponyme ou un adjectif dérivé d'un toponyme, ce toponyme représentant un point du globe, que ce soit une île, un pays, une région ou une partie du monde.

\section{I.2.1. Un rappel historique, élément déterminant}

Les Balkans furent un creuset où se mêlèrent divers peuples soumis d'abord aux Turcs, bientôt convoités par la maison d'Autriche et par la Russie, puis le théâtre de la lutte des peuples balkaniques contre la domination étrangère. Leurs dissensions internes n'ont fait qu'aggraver la situation. La rivalité des grandes puissances et leurs interférences dans la région donnèrent naissance à de nombreux conflits armés, sans parler des campagnes balkaniques et des deux guerres mondiales qui fragmentèrent la péninsule balkanique. L'extension de sens atteint la sphère du figuré avec, pour le substantif, le sème essentiel de "morcellement». Dès lors, le lexique propose à côté du couple morceler / morcellement, plus neutre, le couple balkaniser / balkanisation, vecteur d'une mémoire émotionnelle collective. Ces termes sont devenus courants lorsqu'on évoque ces faits historiques propres, ainsi que des faits apparentés se situant hors des Balkans, dans le monde géopolitique.

\section{I.2.2. Le couple balkaniser / balkanisation}

Selon l'Encyclopeedia Universalis (t. 3, 1990: 756), balkaniser, «semer la discorde et diviser en mini États pour affaiblir» remonterait «au XIX ${ }^{\mathrm{e}}$ siècle, lorsque les nationalités, manipulées par les grandes puissances, s'entre-déchiraient tout en luttant pour leur indépendance». On le trouve attesté en 1917 avec le sens de "faire éclater un pays, un continent, en de nombreuses petites unités, morceler», sous la plume du poète Guillaume Apollinaire, ici journaliste: Toutefois, il ne faudrait pas que l'Allemagne, voyant que son rêve mitteleuropéen ne se réalise pas, parvînt à balkaniser l'Europe. 
[...] Berlin, d'autre part, ne perd pas de temps et [...] cherche à balkaniser l'Europe et avant tout la Russie (Apollinaire, dans Paris-Midi, 19 février 1917, dans CEuvres en prose complètes, III, 479 et 480, Pléiade, 1993, dans Quem. DDL, t. 45). Ils [les Alliés] détruisaient en même temps l'ancienne monarchie dualiste et balkanisaient à plaisir toute la partie de l'Europe centrale située au sud de l'Allemagne ([1938], Edmond Vermeil, L'Allemagne, 13, $2^{\mathrm{e}}$ éd., Gallimard, 1945, Id. Ibid.). Il s'agit d'un concept nouveau, que «morceler» ou "éclater» ne rendent, en définitive, qu'en partie, en raison même de la forte connotation affective, expression de lutte fratricide, de violence, symbolisées de façon paroxystique par le verbe balkaniser qui dispense de tout complément susceptible de rendre cette charge émotionnelle inhérente à certaines situations. Rob.1987 illustre simplement la forme pronominale se balkaniser, «se morceler en de nombreuses petites unités», par Continent qui se balkanise. Le verbe a acquis un sens particulier dans le domaine de la géopolitique.

Il est intéressant de noter également l'emploi participial relevé par Rob.1987: Nations balkanisées par les firmes (extrait du Nouvel Observateur, 22 mai 1978). On peut apprécier l'évolution sémantique de balkanisé retracée par Rob.1987, jusqu'à son statut désormais établi d'adjectif doté du sens "fragmenté, morcelé, divisé, éclaté, atomisé », mais dont les sphères d'emploi laissent aussi percevoir un sens figuré plus marqué: "qui a perdu tout lien le rattachant à un ensemble». Or cette périphrase définitionnelle, extraite du Trésor de la langue française (TLF), révèle toute la richesse sémantique du vocable: La viabilité d'économies balkanisées (Perroux, L'Économie $d u x x^{e}$ s., 1964, p. 341, TLF). On ne trouve pas aisément d'épithète unique synonyme de cet adjectif.

Le substantif balkanisation est attesté dans le TLF par L'Euvre du 27 mars 1941. Le Petit Rober GF0 note sa reprise en 1966, en précisant «à propos d'autres pays » et en soulignant son appartenance au domaine politique, essentiellement avec le sens de morcellement, et Rob.1987 précise: «Éclatement en petites unités nationales d'un territoire jusqu'alors uni par des conditions géographiques ou historiques (à l'image de la diversité des États balkaniques)». Il cite enfin: La balkanisation du continent noir (Le Monde, 1968). Face à l'Europe, à l'Amérique latine et au monde arabe, la politique anglo-américaine a toujours été celle de la balkanisation, du morcellement. Pour dominer ces vastes espaces, innervés par des cultures autochtones pluriséculaires, il fallait diviser pour règner (sic). Il fallait fragmenter, libaniser ( L'ennemi américain », Intervention de Robert Steuckers, 24/11/1991, http://www.nationalbolshevik.com/ synergon/ennemiNov91.htm, interrogation du 19/12/2001). Plus proche encore de nous, l'éditorialiste Jean-François Kahn écrit, de manière prémonitoire: à la fin des fins l'OTAN l'emportera [...]. Le Kosovo (qui aurait pu accéder à une vraie indépendance négociée) deviendra un protectorat américain sans statut, peuplé d'ex-réfugiés sans patrie. Les haines dans toute la région seront devenues inexpiables; les fureurs religieuses et ethniques s'en trouveront exacerbées; dans le monde entier, l'image de l'Occident, réduit à l'Amérique, sera exécrée: la balkanisation forcée appellera des balkanisations désirées (Marianne, 12-18 avril 1999, p. 13).

\section{I.2.3. De nouveaux développements}

Plus récemment, avec l'éclatement en 1991-1992 de la Yougoslavie et l'émergence des problèmes des minorités nationales et des frontières étatiques (guerre en Croatie en 1991-1992, en Bosnie-Herzégovine en 1992-1995, au Kosovo depuis 1999), cette 
région continue d'occuper la scène de l'actualité mondiale. Certains points chauds du globe invitent les journalistes à élargir leur palette lexicale, jouant des toponymes et des suffixes. L'actualité demeure scandée par nombre de menaces et de catastrophes, et les journalistes cherchent dans le nom de tel pays ce qui leur permet de sensibiliser davantage sur la situation de tel autre.

Le verbe se balkaniser est largement utilisé dans la presse politique actuelle, et donne de toute évidence le la à quelques créations à venir: si l'Histoire ne se répète pas à l'identique, elle se plaît souvent à proposer un douloureux «remake» de certains scénarios. Et l'on comprend mieux pourquoi ce terme est toujours en vogue, depuis plus d'une cinquantaine d'années déjà.

Par ailleurs, il est employé dans divers types de situations, les exemples foisonnent, on n'y recourt pas simplement pour filer la métaphore. Force est de constater que ce verbe a acquis droit de cité et a par ailleurs développé une polysémie certaine. Ainsi, en parlant d'autre chose que d'un pays ou d'un continent: A l'UDF, la division est une maladie endémique. [...] lors d'un vote indicatif, les députés centristes ont massivement [...] réaffirmé leur volonté de conserver un groupe parlementaire autonome dans la prochaine Assemblée. Maastricht a par ailleurs consacré l'apparition d'un nouveau féodal [...]. En fait, c'est l'ensemble du paysage politique qui, en quelques années, s'est balkanisé (Le Nouvel Observateur, 8-14 octobre 1992, p. 52).

Outre le sens propre «relatif aux Balkans», l'adjectif balkanisé s'est spécialisé dans le domaine politique, d'où émerge un sens figuré. Cet adjectif a la prédilection des journalistes, notamment pour rendre compte des déchirements et des dissensions des forces politiques, de l'état des partis constitués, et en particulier du parti opposé, dont on déplore le délabrement: Panorama d'une droite balkanisée (titre, Le Nouvel Observateur, 8-14 octobre 1992, p. 52). Autre état des lieux de la France dressé par l'éditorialiste Jean-François Kahn, qui dans un sursaut d'indignation, s'interroge: Hier, les frustrations pouvaient s'exprimer par les urnes: la droite était une alternative. Mais demain, avec un PS de plus en plus balkanisé [...]; [...] un PC encouragé à persévérer dans la surenchère; une extrême droite confortée, et en même temps rejetée dans l'activisme destructeur [...] et une droite si grasse qu'elle attirera les coups de pied comme un gros cul, après avoir pendant cinq ans encouragé et justifié toutes les attitudes inciviques, les violences minoritaires, les violations de la loi...? (Jean-François Kahn, L'Événement du jeudi, 1-7 avril 1993, p. 13).

La consultation des dictionnaires est édifiante. En attendant le verbe balkaniser et son participe passé balkanisé, l'adjectif balkanique, "relatif aux Balkans », qui est y est attesté dès 1886, avait déjà développé, par analogie avec son sens propre, le sens nouveau de «qui est à l'image du morcellement de la péninsule Balkanique» (L'Euvre, 12 avril 1941, dans TLF, extrait de l'IGLF, Inventaire Général de la Langue Française) : «Tragédie balkanique» et «Dunkerque balkanique» sont, du reste, les titres dont use aujourd'hui la presse américaine et d'Amérique latine (id., ibid.).

D'un développement comparable à celui de l'adjectif, le substantif balkanisation prend le sens figuré de «émiettement, dislocation, éclatement d'un ensemble en petites unités indépendantes»: balkanisation dans l'organisation hospitalière française (Le Monde, 31 janvier 1969 dans Rob.1987). Le Dictionnaire de l'Académie, $9^{\mathrm{e}}$ édition, t. 1, 1992 note les sens propre et figuré en illustrant ainsi ce dernier: La balkanisation d'un service. Dans ce même ordre d'idées, on peut lire, à propos des États-Unis, dans le Los Angeles Times: Il n'y a plus de normes nationales pour la culture, le comportement, 
la religion. [...] L'intégration est à présent remplacée, non par l'acculturation, mais plutôt par la balkanisation de la vie américaine: chaque groupe s'imagine que l'autre ne peut le comprendre. Le sens d'une communauté nationale, d'une culture nationale, le ciment qui nous unissait disparaît. Le pays devient un ensemble de petits groupes (L'Événement du Jeudi, 9-15 décembre 1993, p. 66, encadré).

\section{Dérivations néologiques en -iser, -ifier}

\section{II.1. À partir du toponyme}

Sur le modèle de ce couple balkaniser / balkanisation, l'Histoire a fait la fortune de quelques autres couples ou formations isolées.

\section{II.1.1. Le verbe réfère à un conflit violent: massacre, guerre}

Le verbe finlandiser est attesté depuis 1973 dans le domaine politique, au sens littéral de «engager un, plusieurs pays dans un processus de neutralisation (par l'Union soviétique) », l'agent exprimé ou sous-entendu devenant dans certains cas l'idéologie communiste, ou simplement «de gauche »: L'Europe risque, à la fois, d'être "finlandisée» par l'URSS et "latino-américanisée» par les États-Unis (L'Express, 12/11/1973, dans Gilbert, Dictionnaire des mots contemporains, 1980, s.v. finlandisation). Marc Ferro [...] décortique l'affaire (Kravchenko) en s'appuyant sur un témoin de l'époque [...], pilier d'une gauche non communiste, dont il explique très bien à quel point elle fut menacée d'être "finlandisée» (neutralisée) par le PCF (Télérama, 13 janvier 1999, p. 96). Si le lecteur comprend le souci de l'auteur de gloser finlandiser pour plus de clarté, il le suit aussi dans sa préférence pour le nouveau terme par rapport à celui de «neutraliser » : finlandiser, plus évocateur, renvoie de surcroît au système de l'Union soviétique, avec référence, dans le texte, aux moyens que furent les siens, tel le goulag.

Le participe passé devient adjectif: Reagan s'en prend aux Européens mentalement finlandisés, amoureux d'une licorne, la détente (L'Express, 26 juillet 1980, p. 54, dans Rob.1987).

Autre lieu, autre drame, illustré par le couple de termes libaniser / libanisation. Dans un Moyen-Orient en proie à des guerres à répétition, déstabilisantes, le Liban, cerné par la Syrie à l'est et Israël au sud, voit déferler sur son sol un exode palestinien. Des affrontements avec les Palestiniens éclatent de 1970 à 1976. Le Petit Larousse illustré 2001 précise, s.v. Liban: «1976: ils [ces affrontements] dégénèrent en guerre civile; la Syrie intervient. S'affrontent alors une coalition de "gauche» [...] et une coalition de «droite» [...]. 1991: le désarmement des milices et le déploiement de l'armée libanaise dans le Grand Beyrouth et le sud du pays [...] marquent l'amorce de l'autorité de l'Etat, sous tutelle syrienne». S'explique alors, ici ou là, la référence journalistique au drame du Pays du Cèdre, naguère terre d'accueil, nation prospère, pluriethnique et multiconfessionnelle jusque dans les plus hautes instances politiques, et aujourd'hui éclatée, dominée. Ces derniers traits colorent fortement le verbe libaniser, qui en vient à signifier de façon concrète «faire sauter en éclats, éclater»: les dictateurs musulmans de la région [...] font le malheur des Palestiniens, confie l'ancien maire de Jérusalem. "Ils veulent un État palestinien pour nous libaniser, pour nous détruire (Le Nouvel Observateur, 23-29 avril 1992, p. 25). 
L'éditorialiste Jean Daniel, s'interrogeant sur le climat de «corruption » au sein du «personnel politique et administratif de France», fait également allusion au Liban, et, conjointement, à la situation sévissant alors en Italie: on voit [...] que les uns et les autres sont prêts à s'entre-accuser de toutes les [...] forfaitures. [...] Or je sais [...] que, si cette petite guerre de tous contre tous continue dans la société politique et médiatique, [...] le climat va devenir irrespirable et la démocratie dangereusement fragile [...]. Au nom de la morale, c'est la police qui s'installe entre nous. [...]. Lentement, progressivement, inexorablement, nous nous italianisons en attendant de nous libaniser (Jean Daniel, Le Nouvel Observateur, 26 novembre - 2 décembre 1992, p. 56).

Le sens de l'adjectif libanisé(e) «divisé(e), éclaté(e), au terme d'un processus de désagrégation politique et sociale» est directement issu de celui du verbe. Dans la représentation que s'en fait l'inconscient collectif général, la perception du drame libanais peut être associée aux fantasmes propres à chaque individu. Ainsi par exemple se profile, selon tel lecteur du Figaro Magazine, le problème indiscutable de l'islam en France [...]. J'ai même signalé à M. Léotard, qui critiquait le terme d' «invasion» employé par M. Giscard d'Estaing, que nous serions «libanisés» en France si des mesures sérieuses n'étaient prises à temps (Figaro Magazine, 29 octobre 1994, p. 30). JeanFrançois Kahn se demande par ailleurs: Quelque part le corporatisme a eu raison des socialistes. Pourquoi baisserait-il les bras? [...] A moins qu'émerge le monsieur Dupont improbable qui, au lendemain du scrutin [...], osera déclarer aux Français: la comédie est terminée. Nous sommes en charge d'une France libanisée dans un monde en miettes (L'Événement du jeudi, 18-24 mars 1993, p. 9).

En Europe s'éternise le problème de l'Irlande du Nord, dont le rattachement au Royaume-Uni date de 1972, il est l'épicentre d'une agitation endémique faisant du nord de l'île le théâtre d'affrontements continuels entre l'IRA et l'armée britannique. Ce problème s'étend jusqu'en Angleterre et ne cesse de hanter les esprits. Il n'est donc pas étonnant de voir le verbe utilisé à propos du Jura suisse. En effet, créé en 1978, celui-ci englobe trois districts ayant appartenu au Canton de Berne. L'opposition entre autonomistes et pro-bernois se radicalise. Sous le titre Le Jura irlandisé, le journaliste Alain Charpilloz constate en 1976 que le ressentiment et la violence éclatent dans les vallées du Sud, maintenus dans le canton de Berne (Lexique identitaire, Interci$I Z$, interrogation Internet du 04/12/00 ).

La presse a donné la vedette à rwandiser après la tragédie qui a décimé une bonne partie de la population du Rwanda dans un conflit ethnique retentissant. Colonie allemande, puis belge, le Rwanda a souffert d'une grave fracture sociale entre aristocrates Tutsi et bergers Hutu. L'ingérance étrangère inquiétée par les visées politiques des premiers a cédé le pouvoir aux seconds, qui spolient et briment alors les Tutsi. La presse relate cette guerre civile ponctuée de bains de sang en précisant qu'il s'agit d'un génocide. Et c'est ce même terme qui sert par exemple à exprimer l'ethnisation de cet autre conflit en Côte d'Ivoire: Terrribles similitudes (suit une dénonciation circonstanciée, embrigadement de la jeunesse, achat des électeurs [...] qu'est-ce qui a changé par rapport à l'ère Bédié? [...] Le discours exclusionniste et les injures à la communauté internationale continuent. [...] la France [...] qu'on croyait dans sa poche ose dire la vérité, refusant ainsi toute complicité. Il faut donc l'accuser de vouloir «rwandiser» la Côte d'Ivoire [...]. Attention, Mesdames et Messieurs ressaisissezvous! Sinon bonjour le Rwanda! (Patriote archives, Quotidien d'information ivoirien, $\mathrm{n}^{\circ} 327,11 / 08 / 2000$, «Tribune libre», p. 1-3, http://www.lepatriote.ci/pol327-6.htm). 
II.1.2. La transformation évoquée par le verbe est due à une agression d'un type différent

II.1.2.1. La transformation évoquée par le verbe est due à une agression morale et politique

En politique étrangère, en ce qui concerne le soutien des Occidentaux et par allusion à la situation prévalant dans un pays autre que le Congo, en l'occurrence en Russie, congoliser signifie "maintenir au pouvoir (tel homme), contre le gré même et la volonté des élus de ce pays»: Comment Bill Clinton et les dirigeants occidentaux ne comprennent-ils pas ce qu'on entend en Russie lorsqu'ils s'adressent à Boris Eltsine pour [...] l'implorer de "poursuivre, comme on dit, les réformes" [...]. "Réformes", disonsnous, mais quelles réformes? Celles qui ont consisté, depuis six ans, [...] à enrichir les riches, à appauvrir les pauvres, à remplir les coffres off-shore et à "congoliser» la Russie? (Le Nouvel Observateur, 3-9 septembre 1998, p. 44).

\section{II.1.2.2. La transformation évoquée par le verbe est due à une agression de} nature environnementale

À l'instar de la mise en valeur forcenée des Îles Baléares, baléariser signifie « exploiter massivement les possibilités touristiques d'une région au détriment de ses autres ressources économiques et sans égard à la beauté de son site, de son littoral aux fins d'une spéculation effrénée », ce qui peut se résumer d'un mot, "bétonner»: On apprend [...] que des Corses [...] s'apprêteraient à jouer les intermédiaires pour permettre l'investissement sans risque de capitaux étrangers en Corse. Rien de condamnable en soi dans une telle pratique, si ce n'est qu'elle préfigure un développement sauvage de l'immobilier [...]. Le rêve [...] est [...] de baléariser le dernier joyau de la Méditerranée [...]. [...] les trente années de combat qui ont protégé l'île de Corse de la lèpre bétonnière, seraient réduites à néante (Gabriel-Xavier Culioli, dans Journal de la Corse «Autour de la table de Matignon», p. 3, interrogation sur Internet du 28/11/2000).

\section{II.2. À partir de l'adjectif}

Survolant l'espace et le temps, les journalistes créent de nouveaux verbes «télescopiques» qui résument des contextes vécus et vivants.

Ce n'est pas ce type de créations en soi qui est intéressant. Nombreux sont en effet les verbes en -iser d'usage déjà ancien et qui viennent spontanément à l'esprit : américaniser (1855, Quém. DDL dans TLF), angliciser (avant 1757, TLF), franciser (vers 1534, «donner une forme française à un mot étranger», et vers 1698, "donner un caractère français à (qqc)», DHLF, sous français), italianiser (1578 sous la forme italianizer, $H$. Estienne dans TLF), russifier (1830, TLF).

Notre propos est bien plutôt de mettre en lumière un ensemble de néologismes ainsi formés, et qui ont généralement pour sens (a) «rendre» suivi de l'adjectif, ce dernier ayant pour valeur « relatif à tel(le) pays, région, île...» ou encore « originaire de (tel(le) pays, région, île...)»; (b) un sens différent, tout à fait particulier. Intéressants à tous égards - sociologique, économique, culturel, ethnolinguistique, politique ces verbes et leurs contextes témoignent de l'évolution, parfois de la mutation s'opérant sous nos yeux. En tout état de cause, ils s'inscrivent dans une mondialisation de l'information et une prise de parole libérée, caractéristique de notre époque. 
II.2.1. Le verbe désigne « rendre » + l'adjectif, « relatif à tel(le) pays, région, île......»»

II.2.1.1. La transformation évoquée par le verbe concerne l'être tout entier, de façon radicale

L'histoire nous fournit des exemples nombreux, nous en citerons quelques-uns. «Rendre turc», dans le cas de turquiser, prend la valeur de "marquer fortement des traits de la civilisation turque», notamment sous l'égide de Mustapha Kemal: Lorsqu'il adopte le slogan de Ziya Gökalp, "turquiser, moderniser, occidentaliser», Kemal se place dans la lignée des Jeunes-Turcs, dont il est le disciple direct [...]. Le kémalisme entend porter le changement au cœur même de la société, sur la base d'un système politique autoritaire et centralisé, dans lequel le règne du parti unique n'est tempéré que par le paternalisme charismatique d'Atatürc (Encyclopadia Universalis, t. 18, 1990, s.v. Moyen-Orient). Le verbe turquifier, a le même sens: Déçus dans leurs aspirations nationalistes, les Kurdes ont mal supporté d'être intégrés dans la république turque; leurs soulèvements sont suivis d'une terrible répression, qui annihile pour longtemps toute velléité de révolte. Le gouvernement turc s'efforce au maximum de turquifier les Kurdes, mais n'y réussit que partiellement, sous Atatürc).

Le verbe vietnamiser désigne «rendre vietnamien, ici, communiste, par une guerre d'occupation et l'installation d'un régime pro-vietnamien »: Cet ouvrage reflète la politique d'expansionnisme et d'hégémonisme du Vietnam visant à vietnamiser le Cambodge, dans le passé ainsi qu'à l'avenir (Sélection du catalogue du Centre culturel français de Phnom Penh, Nuon Gheun, La marche vers l'Ouest, 1983, consultation d'Internet, Altavista du 15/02/2001).

II.2.1.2. La transformation concerne les luttes qui opposent les pays,

et leur stratégie guerrière

Plus particulièrement, par référence à la politique extérieure des deux superpuissances et aux guerres dans lesquelles elles ont été ou sont encore impliquées, le sens des verbes suivants devient «faire jouer les oppositions entre clans rivaux sur place avec désengagement progressif de la superpuissance étrangère».

Toujours en évoquant la présence russe en Tchétchénie, comme jadis celle de l'Union Soviétique en Afghanistan, et les divisions entretenues dans la région, sous le titre La nouvelle guerre du Caucase on lit: Toutes les tentatives de Moscou pour saper l'influence et la cohésion des indépendantistes ont lamentablement échoué: l'administration tchétchène pro-russe n'a jamais pu acquérir la moindre légitimité [...], et les politiques de division, visant à "afghaniser" la guerre en fomentant une "guerre de clans", n'ont pas réussi à entamer l'unité des Tchétchènes - caractéristique des périodes difficiles (François Jean, Texte publié dans Central Asian Survey - 1997 - p. 413 à 424, interrogation Internet du 18/12/2000).

Tchétchéniser veut dire "rendre tchétchène», pour ce qui est de la Russie, ce verbe signifie plus exactement cf. «contrôler le conflit sans engager de troupes supplémentaires tout en jouant des oppositions déjà existantes sur le terrain»: il est difficile de distinguer civils et combattants dans ce qui est, essentiellement, une guerre de partisans contre une armée étrangère [...]. D’où l'interprétation inévitable entre camps ennemis mal définis: une large "zone grise» est faite de "supplétifs» tchétchènes qui travaillent en réalité pour les indépendantistes; de militaires russes qui passent des accords 
secrets avec les Tchétchènes, ou leur vendent des armes, pour éviter d'être attaqués; et de rebelles, souvent "islamistes", qui sont de vrais agents des services russes. Le Kremlin, sachant que sa présence militaire est au maximum des capacités du pays, tente, depuis des mois, de «tchétchéniser» le conflit (Le Monde, 3 octobre 2000, p. 6).

\section{II.2.1.3. La transformation concerne la langue officielle d'un pays}

Il s'agit toujours de conflit, ici sur le front linguistique, puisque le verbe désigne «contraindre une région, une population donnée à employer la langue dominante comme facteur d'intégration et d'assimilation». Le conflit qui endeuille le Kosovo habité de groupes identitaires différents se joue aussi sur le front linguistique et on saisit l'opposition entre les verbes albaniser et serbiser «imposer comme langue nationale (l'albanais pour les uns, le serbe pour les autres) »: Le HCR note que nombre de membres de la communauté turque kosovare - environ 60000 personnes avant la guerre - sont partis pour la Turquie. Cette communauté est l'objet de pression croissante, notamment de l'Armée de libération du Kosovo (UCK), visant à l' »albaniser» et à lui faire abandonner sa langue, a constaté l'AFP (Revue de presse de l'AUF — Europe orientale, AFP International 30/08/1999. Interrogation internet du 28/11/2000). $S$ 'albaniser, "adopter l'albanais », est attesté infra (v. albanisation).

Serbiser s'entend comme «imposer la langue serbe, notamment aux albanophones du Kosovo, en l'occurrence dans le but de leur assimilation ». UNE LANGUE $B O S N I A Q U E$ ? L'échec de la politique de serbisation. La politique linguistique adoptée par la Serbie au Kosovo en était une d'assimilation. C'est pourquoi elle était appuyée par une politique de serbisation destinée à modifier la composition ethnique de la province. Mais cette politique de serbisation s'est avéré (sic) un retentissant échec. [...] le gouvernement de Milosevic n'a jamais réussi à serbiser les albanophones qui se sont Kosovo, http://www.ciral.ulaval.ca/alx/amlxmonde/europe/Yougserbk.htm, interrogation internet, 18 décembre 2000)

\section{II.2.2. Sens particuliers}

\section{II.2.2.1. Contrôler un gouvernement}

À propos de la Tchétchénie, afghaniser a le sens de «contrôler un pays, un gouvernement, en y suscitant, en y entretenant la division », bref, les diviser pour régner. Il s'agit ici de la Russie et il est fait écho à ce que fut l'occupation soviétique de l'Afghanistan des années 1980, ainsi qu'à la guerre civile et au chaos qui s'en suivirent: Aujourd'hui, [...] personne ne veut un éclatement de la Russie [...]. L'opinion publique a sans doute une vision très négative du comportement russe. [...], la Tchétchénie, livrée à elle-même de 1996 à 1999, a sombré dans le chaos [...]. L'activité des bandes mafieuses et des islamistes a été attisée par Moscou pour afghaniser la république (L'Express, 23 mars 2000, p. 122).

\section{II.2.2.2. Empreindre de la marque du pays d'accueil}

L'objet du verbe asiatiser, «rendre asiatique», désigne un ouvrage emblématique: Les Coréens rouleront dans le Ko Sok Chon Chol (TGV). GEC Alsthom, qui [...] fournira 46 rames (....). Les 34 dernières rames seront fabriquées par des industriels coréens aux termes d'un important accord de transfert de technologies [...]. Nous avons montré aux 
Coréens que, intrinsèquement, nous étions plus aptes à transférer nos technologies que les autres [...]. Nous avons "asiatisé» le TGV! (Challenges, septembre 1994, p. 76).

Si cubaniser a bien le sens de "rendre cubain», une nuance négative s'insinue dans ce verbe, qui évoque en même temps la volonté de restreindre toute autre influence possible (espagnole, américaine, etc.): Trente ans d'athéisme déclaré n'offrent aucune morale de substitution, et le pape entend bien, lors de cette visite pastorale, non seulement revigorer son Église, encourager de nouvelles vocations (qui cubaniseraient davantage un clergé encore très attaché par sa formation, à celui d'Espagne), mais aussi sensibiliser la grande majorité des Cubains au message christique de l'Église (Le Figaro Magazine, 24 janvier 1998, p. 28).

II.2.2.3. La transformation est la conséquence de bouleversements politiques, notamment d'émancipation, d'indépendance

D’après le $G L U$, africaniser signifie «substituer des Africains aux fonctionnaires européens».

Dans le domaine économique, au temps d'Atatürc, turquifier désigne «rendre turques les sociétés étrangères, les nationaliser»: En matière économique, la tâche n'est pas moins grande pour faire de la Turquie un pays moderne et la libérer de la tutelle économique de l'Europe occidentale. Après l'abolition des Capitulations, qui avaient permis aux Occidentaux d'obtenir peu à peu des avantages exorbitants, Mustafa Kemal a travaillé à turquifier, puis à nationaliser les sociétés étrangères, fort nombreuses en Turquie (Encyclopaedia Universalis, t. 3, 1990, sous Atatürc).

Corsiser, c'est, à propos de certaines revendications, «réserver, restreindre aux Corses»: il y a des nationalistes qui distinguent entre Corses et "allogènes "ou qui parlent de "corsiser» les emplois (Charles Lambroschini, Fédération du Parti Radical de Corse, 22 août 2000, Le Monde, interrogation Internet du 18/12/2000). On rencontre aussi son contraire, le dérivé antonymique décorsiser: Veut-on un jacobinisme corse lorsque l'on dénonce, avec justification, le jacobinisme français? Même observation sur la "corsisation complète» de l'administration en Corse. Faudrait-il, dans le même temps, "décorsiser» l'administration continentale? (Guillaume Lenoir, "Quand le problème corse nous saute à la figure...», Unité normande $\mathrm{n}^{\circ} 237$ — Septembre 2000 ; interrogation Internet du 18/12/2000).

II.2.2.4. La transformation évoquée par le verbe a trait à un aspect particulier du comportement des habitants

Cette transformation concerne les habitants d'une région particulière, ou la population dans son ensemble. On note, à propos du centre de la France, et, par analogie avec la situation en Corse, la spécialisation de sens du verbe se corsifier, «se maintenir dans l'indivision en évitant l'ouverture d'une succession reçue en héritage entre parents éloignés »: Le Conseil supérieur du notariat pousse un cri d'alarme: la France se corsifie! Corsification, un mot barbare qui se réfere à la situation de la Corse où les héritiers ont repoussé de génération en génération l'ouverture de leur succession. A l'origine de ce maintien de l'indivision, on trouve le coût des droits d'enregistrement, jugé prohibitif, voire confiscatoire pour les petits héritages entre parents éloignés (Investir $\mathrm{n}^{\circ}$ 682, Supplément, 14 février 1987, p. 48).

S’italianiser figure dans le Dictionnaire de l'Académie ( $9^{\mathrm{e}}$ édition, 1999, fascicule $\mathrm{n}^{\circ} 2$ ) avec le sens de «adopter des coutumes, des manières italiennes ». Dans l'article 
de Jean Daniel (Le Nouvel Observateur, 26 novembre-2 décembre 1992, p. 56, cité supra à propos de se libaniser), ce n'est pas un néologisme de sens à proprement parler, puisqu'il signifie «se conduire comme le font les italiens », clin d'œil du journaliste à l'opération «mains propres (mani pulite)», menée contre la mafia. Dans ce contexte on est frappé par la brochette de termes employés par l'auteur: corruption, forfaitures, perversion, prébendes, malversation, qui font simplement écho au substantif sicilianisation cité infra.

\section{Création de substantifs féminins, dérivés en -isation / -ification}

Ces substantifs sont créés soit directement à partir de toponymes, soit à partir d'adjectifs issus de toponymes. La plupart des créations substantivales forment un couple avec le verbe originel, comme le modèle balkaniser / balkanisation, avec substitution de suffixe: -iser / -isation; -ifier / -ification.

Il y a lieu d'étudier dans un premier temps finlandisation, qui, comme balkanisation, est traité dans les deux dictionnaires de langue du xx ${ }^{\mathrm{e}}$ s., Rob.1987 et le TLF — ou, plus exactement, ce qui devait initialement devenir son Supplément et à la nomenclature duquel nous avions suggéré l'ajout du couple finlandiser / finlandisation, seul le substantif ayant alors été retenu.

III.1. L'étymon désigne un nom de pays, ou d'état, encore en usage ou non

\section{III.1.1. Les «formations » finlandisation et libanisation}

Il s'agit dans le cas de finlandisation de la «neutralisation (d'un pays, d'un ensemble de pays) par l'Union soviétique»: La finlandisation de l'Afrique, de la République fédérale d'Allemagne (Rob.1987). Clef de voûte de ses relations avec Moscou et de sa politique étrangère, c'est l'objet historique de la sempiternelle référence à la « finlandisation", que l'on préfere ici traduire par «neutralité active» (L'Express, 7 juin 1980, p. 112, ibid.).

On a cru, dit Minc, que l'harmonie démocratique allait succéder à l'ordre communiste et que les Occidentaux, forts de leur victoire historique, pourraient réguler un monde réconcilié. Ce fut une grande naïveté (Minc [...] avait, lui, annoncé, il y a quelques années, la finlandisation de l'Europe par la puissance soviétique) (Laurent Joffrin, Le Nouvel Observateur, 6-12 janvier 1994, p. 76).

Avec un glissement de sens, sans référence explicite à l'Union soviétique mais avec l'idée sous-jacente de "pressions politiques» issues de courants «de gauche», on lit: L'université est menacée de politisation, d'une sorte de finlandisation aux conséquences désastreuses (Libération, 8 décembre 1983, dans Rob.1987).

Le Dictionnaire de l'Académie, dans sa $9^{\mathrm{e}}$ édition, propose pour tout article le seul référent historique du terme: «Dérivé de Finlande, sur le modèle de balkanisation. Polit. Expression usitée pendant la période dite de "guerre froide" (1947-1989) pour qualifier la neutralisation de pays européens qui, comme la Finlande, étaient soumis à de fortes pressions de la part du bloc oriental, sans adhérer formellement au pacte de Varsovie». Aucun sort n'y est donc réservé au verbe de même racine, pas plus qu'au verbe dont est issu le modèle balkanisation. L'Académie fait dériver les substantifs balkanisation et finlandisation directement du toponyme correspondant, et pourtant, ces verbes ne sont-ils pas incontestablement attestés, avec une avance de trois décennies en tout cas pour celui qui sert de modèle: balkaniser? 
Outre la transformation historico-politique subie par le Liban, le substantif libanisation suggère l'idée générale de l'«éclatement sanglant d'un pays (au terme d'une guerre civile entre communautés de races et de religions différentes)»: L'Afghanistan n'est plus en tête des priorités politiques de Washington. [...] La guerre peut donc durer encore si longtemps que la «libanisation» du pays, son enlisement dans les massacres, soit irréversible (L'Evénement du jeudi, 19-25 avril 1990, p. 10). Toujours hors du contexte libanais, on relève, à propos de la Bosnie-Herzégovine, le syntagme candidat au figement logique de libanisation: Le compromis proposé [...] est celui de la "cantonalisasion» de la Bosnie-Herzégovine, à la manière helvétique. Compromis discuté et pourtant favorable aux Serbes [...]. Ce compromis, même s'il contribue à ce que Joseph Kulic appelle la «logique de libanisation» de l'ancienne Yougoslavie, est probablement la seule manière d'arrêter la guerre, pour le moment (Jean Daniel, Le Nouvel Observateur, 14-20 janvier 1993, p. 38).

Le sens passe de "déchirement dont souffre une région revendiquée par des composantes ethnico-religieuses différentes» à celui de "lutte», voire à celui de "guérilla», comme dans l'extrait suivant: Ambiance de deuil et de colère en Israël et dans les Territoires palestiniens. [...] la «libanisation» est bien là. Le mot désigne la guérilla menée au Liban-Sud au temps de l'occupation israélienne. A coup d'embuscades et de bombes télécommandées, les islamistes chiites du Hezbollah ont fini par entraîner le départ de l'armée israélienne, qui a décidé que le prix était trop lourd (sous le titre: cf. «La Cisjordanie en voie de «libanisation», Le Figaro, 15 novembre 2000, p. 3).

\section{III.1.2. Les autres formations}

III.1.2.1. Le substantif exprime un processus relatif à un conflit violent

Le déchirement sanglant de la Bosnie rendue célèbre par les attentats terroristes perpétrés dès 1992, par le siège de sa capitale Sarajevo, l' «épuration ethnique» de sa population, les bandes de «snipers» s'arrachant la une des journaux, fait du néologisme bosnisation la meilleure évocation d'une soudaine transformation en "poudrière", d'un état d'insécurité et de menace perpétuelle: Pour Uri Dan, Israël est en voie de "bosnisation», titre sur deux pages, Le Figaro Magazine du 2 mars 1996 (p. 22-23), et, poursuivant à la page 23, ce journaliste précise sa pensée: Nous entrons dans une phase de "bosnisation" d'Israël. La violence terroriste va s'accroître dans la mesure où armes, munitions ou explosifs comme le TNT s'entassent dans les villes contrôlées par les Palestiniens (id. ibid.).

En ce qui concerne le Jura suisse et la menace pesant sur le français dans cette région, ce qui n'est qu'une composante d'une révolte plus générale, l'association intitulée «Association romande pour la défense de la langue française » ávoqué en 1975 la «Menace sur la frontière des langues», n'hésitant pas à parler de l' «irlandisation» du Jura à propos du problème linguistique que soulève précisément la germanisation de cette région (v. Interci-IZ, www.synec-doc.be/francite/lexid/Ixid403.htm, interrogation Internet du 4/12/2000). En vérité, cette «irlandisation» y est la résultante de diverses composantes tant politique, économique, sociale, culturelle que religieuse à l'instar de la situation qui règne en Irlande du Nord.

Les atroces massacres entre Hutu et Tutsi, perpétrés au Rwanda, théâtre à ce jour d'affrontements intercommunautaires permanents, font que le dérivé rwandisation concentre tout ce qui a trait à un génocide, jusqu'à devenir le synonyme même de ce 
terme. En afrilangue génocide peut se dire «rwandisation» ou «somalisation» (Actualité $d u$ FLE, www.fdm.hachette-livre.fr/actus/000301.html, interrogation Internet du 28/11/2000)

III.1.2.2. Le substantif exprime un autre type de transformation, notamment d'ordre politique, administratif, social, économique ou culturel

En politique, bénéluxisation désigne le "passage sous le contrôle des autorités du Bénélux» (Dictionnaire des termes nouveaux des sciences et des techniques, CILF 1983).

Le substantif, tel congolisation, désigne souvent le fait de permettre à des autochtones de s'approprier des usines, des sociétés: En dehors de toute prise de position d'ordre politique et à titre personnel, je regrette la décision du Ministre Congolais des Postes et Télécommunications [...] de nationaliser les parts de Mr. Mikp Rwiyitare dans la société Telecel. Hier Belges, aujours'hui Rwandais, demain ... ? Hier Zairianisation, (sic) aujourd'hui Congolisation, demain ... ? Par rapport à une activité industrielle telle que les telecoms, où les initiatives d'investissement doivent pouvoir s'inscrire dans un plan financier construit sur le long terme afin de faire sortir de terre des infrastructures modernes faisant entrer tout Congolais dans la société d'information, cette initiative est malheureuse (Nationalisation partielle de Telecel, Eric A. Smekens, http:/www. congoline.com/ Forum1/Forum00/ Smekens03.htm).

La corsisation ou corsification est la "réattribution des emplois corses à des insulaires»: Apprentissage obligatoire du corse à l'école, création d'une université à Corte, «corsisation» des enseignants [...] les revendications des autonomistes corses sont en grande partie culturelles (D. Dhombres, in Le Monde de l'éducation, oct., II. 1975, Mots nouveaux contemporains 1, 1993, Publications de l'INaLF). Le journaliste Patrick Poivre d'Arvor, recevant Henri Emmanuelli au journal de $20 \mathrm{~h}$ le 12 novembre 2000, lui demande à propos de la Corse: - la langue corse obligatoire aboutirait à la corsification des emplois?

Avec une spécialisation de sens, et par analogie avec la situation en Corse et à propos du centre de la France, c'est le «maintien dans l'indivision en évitant l'ouverture d'une succession reçue en héritage, entre parents éloignés »: Corsification, un mot barbare qui se réfère à la situation de la Corse où les héritiers ont repoussé de génération en génération l'ouverture de leur succession. [...] Surtout, des patrimoines entiers tombent en jachère, le cadastre perd toute valeur et les opérations de remembrement ou d'expropriation ne peuvent plus être menées faute de propriétaires connus. Le centre de la France est le plus touché, mais ce phénomène s'étend de jour en jour (Investir, Supplément, op. cit.). Cette dernière attestation montre l'implantation de diverses valeurs de ce terme, comme c'était le cas du verbe se corsifier, supra. Nous suggérions déjà (en vain) l'entrée de ce couple dans ce qui devait devenir le Supplément du TLF.

Par référence à la toute récente union de l'Europe, l'européisation est la «prise en compte de la suggestion d'un État-membre de l'Europe, pour résoudre une crise politique intérieure au sein de l'un d'entre eux (l'Italie, dans le cas d'espèce) »: Lionel Jospin au secours du gouvernement Prodi... Une "solution à la française» de la crise italienne... L'européisation des politiques va décidément bon train, depuis que les choix de Paris sont devenus un enjeu de politique intérieure à Rome et que la voie française a permis de résoudre, en Italie, "la crise la plus folle» —, celle que Refondation communiste avait ouverte il y a dix jours en retirant son soutien à la coalition de centre-gauche du Premier ministre Romano Prodi (Le Nouvel Observateur, 16-22 octobre 1997, p. 61). 
Au Maroc, la marocanisation est la "prise de contrôle d'une entreprise par des responsables marocains» (GDT, source de 1974).

La saoudisation désigne l'embauche obligatoire de Saoudiens: Sous le titre «Les entreprises françaises trop timides en Arabie Saoudite», le journaliste détaille entre autres difficultés Les obstacles occasionnés par les spécificités du pays : fermeture de 30 minutes cinq fois par jour à l'heure des prières, corruption, manque de formation de la main-d'cuvre locale. Et la 'saoudisation' de cette dernière aggrave encore la situation. Contraintes d'embaucher chaque année 5\% de Saoudiens en plus, les entreprises sont confrontées à un personnel constitué 'de gens qui ont très peu de bases et dont il est difficile de rehausser le niveau' (Le Figaro Économie, 12 février 2001, III, p. 37, encadré).

Par référence à la Sicile, mais avec une originalité morphologique, le terme sicilianisation désigne le "régime de non-droit dû à une dérive maffieuse»: Aujourd'hui, ce qui menace l'Europe, c'est une régression pathologique de l'Etat: non seulement de l'Etat-nation, mais de l'Etat de droit. [...] En fait, ce qui menace désormais l'Europe, ce n'est plus la "balkanisation» ni même la "libanisation", c'est la sicilianisation, le règne des parrains, des seigneurs de tous ordres (Globe Hebdo, 21-27 avril 1993, p. 13).

L'action de rendre turc, c'est la turquisation: En 1909, la doctrine de "turquisation intransigeante", mise en application de façon systématique par les "Jeunes Turcs" arrivés au pouvoir l'année précédente, causera déjà la mort de 20000 Arméniens (Séance du 7 novembre 2000, Sénat, Travaux parlementaires, comptes rendus, www.senat.fr, interrogation Internet du 22/02/2001). Turquification a le même sens: Les premiers militants arabistes adhèrent au mouvement des "Jeunes-Turcs» dans l'espoir d'accéder à une autonomie politique arabe dans l'empire. Mais, une fois au pouvoir en 1908, les Jeunes-Turcs, animés par un nationalisme turc virulent, travaillent au contraire à une «turquification» de tout ce qui reste de l'empire ottoman (Encyclopoedia Universalis, t. 18, 1990, s.v. Proche et Moyen-Orient contemporain).

La vietnamisation est le fait de «rendre vietnamien»: L'Indochine est successivement le théâtre d'une guerre de décolonisation avec Ho Chi Minh, qui se termina en 1954 par le départ des Français et la vietnamisation du pays (Sélection du catalogue du Centre culturel français de Phnom Penh, Nuon Gheun Les trois guerres d'Indochine, Gilles Férier, 1993, consultation d'Internet, Altavista du 15/02/2001).

III.1.2.3. Le substantif exprime une transformation

de nature environnementale

Aux Îles Baléares dont le tourisme constitue la principale ressource avérée, la baléarisation est l'«exploitation forcenée des possibilités touristiques d'une région au détriment de ses autres ressources économiques »: Sur le continent, on rit de cette île où l'on fait voter les morts. En Corse, on ne rit pas ou on rit jaune. Dans son cabinet du boulevard Paoli, à Bastia, Edmond Siméoni pointe du doigt les projets touristiques mirobolants qui font redouter la "baléarisation» de l'île [...] (L'Express, 12 février 1998, p. 28). Toujours avec ce même sens, à propos de tel autre site touristique: «Je suis seule et je veux le rester", le rosé fait son effet et on apprend enfin ce qu'elle fabrique à Palmiye (Turquie). "Un mémoire sur la baléarisation de la côte méditerranéenne» [...]. »La baléarisation, c'est quand on met du béton partout» (Cosmopolitan, juillet 1991, p. 72). 


\section{III.1.2.4. Le substantif exprime une transformation de nature culturelle}

La hollywoodisation correspond au «fait de marquer de l'influence culturelle américaine (par le truchement de Hollywood, le principal centre de l'industrie cinématographique et de la télévision aux États-Unis)»: Interviewé par L'Express: - La France subit-elle l'influence culturelle américaine? Jérome Charyn: - Le problème ne se pose qu'en termes économiques. Il n'y a pratiquement plus de culture aux États-Unis, hormis Disneyland, E.T. et la hollywoodisation du monde. C'est une industrie. Et ses productions ressemblent à de l'électricité dans l'air, elles n'ont plus de consistance (L'Express, 7 octobre 1993, p. 82).

Par référence à l'extrême exiguïté des surfaces habitables, aggravée au Japon par la surpopulation, japonisation désigne la marque de l'influence japonaise en l'occurrence sur l'habitat français: La «japonisation» des mètres carrés. On distingue peu d'idées directrices dans cette décennie 1990. Néanmoins, il existe déjà une tendance nette à la "japonisation des mètres carrés». Globalement, les pièces sont plus petites et il y en a moins. Même le salon, qu'on aime double, devient un peu plus compact. Les chambres, elles, sont «minimalistes» (Le Figaro Magazine, 23 avril 1994, p. 140).

\section{III.1.2.5. Le substantif exprime une transformation de nature linguistique}

Ce phénomène s’inscrit dans une politique de conquête dans laquelle la population dominée doit adopter pour son intégration, sa survie, la langue du pays dominateur.

L'extrait suivant concerne l'adoption de l'albanais au Kosovo. La victoire des Turcs en 1389 a vu le départ de la population slave-orthodoxe et slave-catholique. Si l'on se fonde sur des données disponibles sur Internet (Libération, Forum sur la guerre en Yougoslavie), on apprend que le reste de la population slave - slavebogomile - n'a pu demeurer sur ses terres qu' "au prix d'une islamisation (très relative, mais obligatoire), ainsi que la population albanophone encore présente depuis le $\mathrm{vI}^{\mathrm{e}}$ s. malgré l'invasion slave»: Le passage du Kosovo slave-bogomile sous domination turque a rapproché ses habitants des Albanais, également islamisés et l' «albanisation » a ainsi pu s'effectuer. Un changement de langue (du serbo-croate à l'albanais) n'est pas une chose impossible en plusieurs siècles, à partir du moment où il y a eu changement de régime politique et de religion (Un Slave peut s'albaniser en 6 siècles, 11.06.99, 17h50, http://www.liberation.com/forums/kosovo/rr2021.html, interrogation Internet du 23/01/2001).

Le pouvoir central serbe a tout tenté pour freiner le séparatisme et corrélativement l'albanisation du Kosovo, et exercer une pression considérable pour promouvoir la serbisation des albanophones (v. serbiser supra).

\section{Points de vue de lexicologue, de lexicographe, de terminologue}

Les définitions parfois encyclopédiques que nous avons proposées permettent de situer les concepts à l'intérieur d'un domaine historico-politique nuancé et précis. Nous avons eu besoin de commentaires pré-définitoires, qui, dans un dictionnaire de langue, seraient susceptibles de prendre place entre crochets ou parenthèses, et qui peuvent rendre compte du choix du journaliste de telle unité plutôt que de telle autre car incontestablement plus vivante, nourrie "de chair et de sang», capable de faire mouche dans un contexte de narcose sociale. Comme par exemple finlandiser qui est 
utilisé plutôt que «neutraliser», balkanisation plutôt que "morcellement», libanisés plutôt que "divisés». En toile de fond se profilent, pour finlandiser, une idéologie pure et dure, pour balkanisé, une déliquescence, voire la disparition, de liens autrefois tangibles, pour libanisés, l'évocation d'un «big bang» sanglant, d'un éclatement jadis insoupçonnables.

En vérité, si le repère historique ayant présidé à la naissance de ces termes venait à s'estomper, le sens n'en deviendrait-il pas soudain obscur ou simplement flou? Les auteurs de ces créations, ainsi que leurs réutilisateurs, sentent que l'exercice est quelque peu "périlleux", aussi se protègent-ils derrière des italiques, des guillemets, des quasi-synonymes parenthésés. Ces "précautions d'emplois» sont nombreuses dans les contextes relevés, et si les auteurs ont recours à des guillemets, c'est que le terme n'est pas, ou pas encore "officialisé», qu'il n'existe pas vraiment, à moins qu'il ne s'agisse d'emplois ou d'occultations euphémistiques tout simplement.

C'est dans le sens de l'hésitation, de l'insécurité linguistique, discursive, pratique, que va l'hésitation des dictionnaires relevée, notamment dans les différentes versions bilingues d'un même dictionnaire. Dans la partie français-anglais du Robert \& Collins, on note que l'anglais to Balkanize est marqué «pol.» seulement dans la partie anglaisfrançais. Pour le français libanisation marqué «pol.» on peut lire l'exemple forgé sans équivalent anglais: "La libanisation du pays, the fragmentation of the country».

Si on comprend d'une certaine manière l'accueil réservé des dictionnaires à l'égard de telles formes nouvelles, on comprend aisément, à l'ère de la mondialisation de la communication, l'accueil enthousiaste qui leur est fait sur bien des sites Internet.

Les unités lexicales étudiées n'ont trouvé place que rarement dans les dictionnaires de langue. C'est qu'elles ont du mal à trouver droit de cité, un peu comme certains emprunts à l'anglo-américain, peut-être en raison d'une trop lourde charge connotative. L'usage de dérivés à partir de toponymes serait en effet à rapprocher du mécanisme de l'emprunt linguistique. On emprunte des objets — au sens large du terme - dont la conceptualisation se fait dans leur milieu d'origine, et qu'on dénomme par la suite. En l'occurrence, l'objet est une image indélébile, une tragédie récurrente dont l'épilogue est le «morcellement» pour le mot phare «balkanisation».

On relève finlandiser et finlandisation dans Rob.1987 alors que seul le substantif se trouvait dans la nomenclature prévue du Supplément au TLF. Pourquoi pareille lenteur? Est-ce en raison de leur apparition relativement récente, ou, pour bon nombre d'entre elles, de leur appartenance à un domaine «sensible»?

Surprises, au départ, par des emplois récursifs de certains dérivés de toponymes, nous avions voulu vérifier l'usage qu'en faisaient les dictionnaires et encyclopédies. Nous l'avons trouvé restreint, ce qui était compréhensible: d'une part, le sème -iser, -isation dans le domaine de l'histoire de la langue est déjà bien connu, mais, surtout, naturellement compréhensible, ce qui permet aux dictionnaires de faire l'impasse sur une partie de ces formations potentielles. D'autre part, les formations récentes que nous avons analysées pouvaient finalement, de la même manière, donner lieu à de longues listes de formations, ce qui n'est pas vraiment intéressant pour les dictionnaires de langue. Ceux-ci ne pouvaient se permettre le luxe d'attester toutes les formes potentielles offertes par un mode de dérivation bien rodé. La prolifération des néologismes substantivaux n'a rien d'étonnant car plus généralement les substantifs dérivés «ne cessent de se multiplier grâce à leur aptitude supplémentaire à se greffer sur les verbes, les noms et tous les types d'adjectifs» comme invite à le constater 
(Quémada, 1978: 1197, citation que nous avions déjà rappelée à l'article -TION, -SION du TLF).

Le nombre d'attestations relevées dans la presse nous a vraiment permis d'appréhender un ensemble de nouvelles valeurs sémantiques pour ces suffixes.

Ce sentiment a été extrêmement amplifié lors de la consultation d'Internet. Nul doute que nous avions effectivement contribué au repérage d'un nouveau sème lié à des évènements politiques nettement identifiables eux-mêmes.

Nous sommes fondées à parler de "termes», relevant de domaines de prédilection: histoire, géographie, politique. Nous nous trouvons bien dans un certain cadre terminologique, mais proches des sphères de la terminologie institutionelle, comme celle qui est parfois traitée par les Commissions spécialisées de terminologie et de néologie. Une des tâches de la Commission de terminologie des Affaire Étrangères ${ }^{1}$ est par exemple l'étude des désignations françaises actuelles des pays et de leurs capitales, des lieux de résidence des consulats français, ainsi que des gentilés. Nul doute que des dérivés bien implantés légitimisent la forme de leur base, le toponyme. Nous ne citerons qu'un exemple: vietnamisation apporte, avec vietnamien, un argument de poids au maintien de la forme canonique du toponymeVietnam qui connaît pourtant 9 variantes en français, selon une étude d'Ange Bizet ${ }^{2}$.

Mais nous avons aussi illustré la polysémie de certaines de ces unités lexicales. Or, à regarder de plus près le contenu sémantique de ces termes, on leur attribue une triple valeur, due à un cheminement en trois étapes, à trois niveaux:

(a) le dérivé a une valeur historique, partant d'une réalité tangible, hic et nunc, dans l'espace et dans le temps;

(b) par analogie de sens, il acquiert une seconde valeur: comme nous l'avons montré dans le premier exemple décrit — notre exemple tête de liste —, le dérivé toponymique, finalement saturé de sa valeur symbolique initiale référant à des faits géographiques et politiques précis, en vient naturellement à exprimer des faits analogues, mais qui se déroulent en un autre lieu;

(c) parfois, au figuré, il prend appui sur ce nom de lieu, non plus pour évoquer un point géographique tel une île, une région ou une partie du monde (physique), mais pour définir une caractéristique ou pour parler d'un fait relatif à une entité réelle que constitue un secteur de la vie sociale, culturelle, économique ou politique. Par exemple, en puisant dans nos citations: économies balkanisées et balkanisation d'un parti politique; (Européens) mentalement finlandisés et finlandisation de l'université.

Il n'y a pas lieu ici d'évoquer l'objection qui pourrait naître d'un rapprochement inévitable entre polysémie des mots de la langue commune et monosémie des termes. Il s'agit plutôt d'un ensemble de traits qui s'organisent en une structure notionnelle appréhendant une réalité définie qui s'inscrit dans un ou des lieux, et qui permet des relations de type «logique» fondées sur la ressemblance, l'analogie — par opposition au sens propre fondé sur «la contiguïté ou le contact dans l'espace et le temps» (v. à ce sujet le rappel de Cabré 1998: 88) mais à propos des types de relations liant les concepts entre eux à l'intérieur d'un même domaine.

En tout état de cause, les termes étudiés sont, à l'origine, des termes de journalistes. Pour en revenir au domaine de la communication qui nous concerne - au travers d'un article de presse par exemple - nous savons que l'émetteur et le récepteur ont en partage, outre la langue, une certaine connaissance de la réalité évoquée; le récepteur - lecteur aura désormais le loisir de reprendre à son compte le terme, avec 
à l'esprit l'équation selon laquelle «notion» et «terme» constituent les deux pôles de la base d'un triangle dont le sommet serait le toponyme.

\section{Synthèse et conclusion}

Tous les dérivés substantivaux étudiés ne connaîtront certes pas tous la même fortune. En revanche, certains de ceux qui ont développé un sens quelque peu nuancé, par extension ou au figuré, ont une plus grande chance de s'implanter. Les dérivés des noms de lieux ont été investis d'un sens paroxystique, une manière de quintessence, si on les compare à tout hyperonyme.

Depuis le couple modèle balkaniser / balkanisation un schème mental s'est frayé un chemin dans l'inconscient collectif, faisant ressortir une référence à un lieu de drame, ou à l'origine d'une discorde. Tout locuteur qui a besoin d'évoquer le concept de «morcellement» ou de «neutralisation» ou encore «d'éclatement» peut aussi bien recourir aux hyperonymes «morcellement» ou «éclatement» qu'aux dérivés de toponymes étudiés dans ces pages.

Celui qui a vu et vécu par procuration la violence qui a endeuillé la Bosnie comprend l'effarement d'un Israélien redoutant la bosnisation de son pays, ou d'un Congolais face à la rwandisation de son pays. La marocanisation a pu chagriner l'industriel français demeuré au Maroc après l'indépendance de ce pays. A l'inverse, la Tchétchénie se voit afghanisée par une guerre de clans de la même manière que l'Afghanistan avait eu à en souffrir par l'intrusion soviétique. Plus récemment, les Russes désespèrent du changement radical intervenu dans leur pays après la chute du mur de Berlin, puisqu'ils se sont vus imposer la dictature d'un chef aveugle à leurs souffrances quotidiennes. Ce sont là les avatars de l'Histoire au passé et au présent. Pour ces derniers, leur passé «glorieux» de «superpuissance» est ravalé au sort d'un pays africain comme le Congo par exemple, d'où l'emploi du verbe congoliser.

L'âpreté des combats économiques rappelle la stratégie guerrière, ainsi par exemple lorsqu'il s'agit, simplement, de remporter un contrat: les Français ne seront pas peu fiers d'avoir asiatisé le TGV. La formation s'italianiser renvoie à un aspect ponctuel de la vie en Italie, la référence étant faite à l'épisode «mains propres»pris en mauvaise part — en relation avec la Maffia. On fait naturellement le rapprochement avec le cas de sicilianisation qui relève du même contexte, la Sicile étant le véritable fief de la Maffia. Les termes sont révélateurs de luttes, de rivalités, de tensions. La balkanisation des services d'un hôpital est plus que regrettable et constitue une entrave sérieuse à la coordination indispensable en pareille enceinte puiqu'elle met en péril la sérénité, le calme attendus. C'est tantôt un constat d'échec, tantôt un cri d'alarme, le sentiment que les choses sont allées trop loin: la finlandisation de l'Université qui ne doit pas être un forum politique. C'est ce que pense, nous semblet-il, sans le dire expressément, mais en le suggérant, l'auteur du propos. Quand un promoteur immobilier obtient un permis de construire pour un complexe immobilier sur le littoral méditerranéen, corse ou turc, cela alarmera naturellement les riverains, mais aussi les écologistes et tout amoureux de la nature choqué par de tels procédés, et tous maudiront cette baléarisation, où qu'elle se produise.

Il y a bien, avec japonisation, cette notion d'exiguïté pénible que la publicité s'empresse en vain de parer d'un charme oriental, ou japonais, et qui constitue un 
écueil réel mais insurmontable, étant donné le coût élevé de ces mètres carrés et les possibilités modestes des acquéreurs.

De ces créations naît un sentiment d'effroi (bosnisation, irlandisé / irlandisation, libaniser / libanisation, rwandisation, tchétchéniser / tchétchénisation), d’inquiétude (corsifier et corsiser / corsification et corsisation, hollywoodisation, s'italianiser, sicilianisation), de rejet (baléarisation, congolisation, serbisation, vietnamisation), sentiment vérifié par les contextes retenus. Cela laisse l'impression que ces termes ne sont pas neutres, qu'ils sont le plus souvent pris en mauvaise part par celui qui subit l'action du procès, la tyrannie de l'un de ses protagonistes ou en raison de l'arme employée. Seules deux ou trois formations semblent non marquées par une réprobation ou par un regret: il s'agit de asiatiser du côté verbal et de bénéluxisation et européisation du côté substantival. Pourtant, on pourrait, pour asiatisé par exemple, surprendre l'idée d'une âpre lutte telle qu'elle est perçue par les vainqueurs, en l'occurrence la firme Alsthom et le TGV. Dans le cas de européisation, il y a eu, certes, en amont, un combat qui a divisé l'opinion et qui a porté le nom de Maastricht.

Ces suffixes sont largement illustrés par des exemples bien souvent de première main, prouvant à l'envi qu'il y a confrontation entre deux points de vue. Le journaliste adopte généralement celui qui lui paraît juste, de façon objective, mais le fait d'écrire pour tel magazine plutôt que tel autre lui permet d'être fidèle à ses opinions politiques: on parlera aussi bien d'une droite balkanisée que d'un PS balkanisé.

Rappeler le lien existant entre l'histoire et les toponymes - par le biais de la géographie - a été nécessaire à l'étude d'une catégorie de nouveaux vocables, plus précisément de nouveaux dérivés de toponymes. Et l'on constate qu'en termes de communication, et, comme on l'a souligné, de mondialisation, ces unités lexicales ont pour mérite de réduire les distances en rapprochant régions et continents, de maintenir en éveil l'esprit du récepteur (de tout message), d'éveiller sa curiosité tant géographique, historique, que linguistique, puisque dans sa vie quotidienne peuvent intervenir de ces vocables aux racines à la fois lointaines et proches.

L'étude morphosémantique de ces quelques termes a permis de souligner le caractère productif des suffixes -iser, -isation, -ifier, -ification, éléments éminemment dynamiques du français, notamment dans le domaine de la politique et plus précisément au croisement de la politique et de la toponymie.

\section{NOTES}

1. Créée en septembre 1999; D. Candel en est d'ailleurs membre.

2. Étude de 1996, proposée à la Commission des Affaires étrangères.

\section{RÉFÉRENCES}

Cabré, M.-T. (1998) : La terminologie, théories, méthodes, applications, traduit du catalan (1992), adapté et mis à jour par M. C. Cormier et J. Humbley, Les Presses de l'Université d'Ottawa, Armand Colin.

QuémadA, B., éd.: Datations et documents lexicographiques, CNRS, Klincksieck, INaLF (ci-dessus: "Quem. DDL»).

QuémadA, B. (1978): Histoire des techniques, Encyclopédie de la Pléiade, p. 1146-1240.

QuémAdA, B., éd. (1993): Mots nouveaux contemporains, Matériaux pour l'histoire du vocabulaire français, CNRS, Klincksieck, INaLF, 1. 


\section{Dictionnaires et encyclopédies, bases de données informatisées}

Le Grand Dictionnaire Terminologique, Office de la langue française, Gouvernement du Québec, 2000 (ci-dessus «GDT»).

Le Petit Larousse Illustré, 2001.

Gilbert, Dictionnaire des mots contemporains, 1980.

Petit Robert Grand format, 1996 (ci-dessus «Petit Rob. GF»).

Robert \& Collins super senior français-anglais, anglais-français, 1995 (ci-dessus «Rob. 1987»).

Supplément au TLF (non publié).

Grand Larousse Encyclopédique (ci-dessus «GLU»).

Trésor de la langue française (1971-1994), Dictionnaire de la langue du $19^{e}$ et $20^{e}$ siècle, CNRS, Klincksieck, Gallimard (ci-dessus TLF).

Dictionnaire de l'Académie, 9e édition.

Encyclopaedia Universalis, 1990.

Quémada, J. (éd.), Dictionnaire des termes nouveaux des sciences et des techniques, CILF 1983.

\section{Corpus de presse}

Challenges, Globe Hebdo, Investir, Le Figaro, Le Figaro Économie, Le Figaro Magazine, Le Monde, Le Nouvel Observateur, L'Événement du jeudi, L’Express, Libération, Marianne, Télérama.

\section{Consultations de dictionnaires et textes divers sur Internet}

Moteur de recherche Google, plus rarement Altavista: les références précises ont été indiquées dans le texte. 